\title{
In spregio o in sfregio? Qualche chiarimento in merito
}

\section{Vittorio Coletti}

PUBBLICATO: 09 LUGLIO 2019

\section{Quesito:}

Alcuni lettori ci sottopongono le due locuzioni a/in spregio e a/in sfregio che benché diverse sembra possano avere usi "pressoché equivalenti"; in una richiesta in particolare si fa riferimento all'espressione usata su Facebook "mettere like a sfregio". Una lettrice poi ci chiede se sia preferibile usare in spregio di qualcosa o in spregio a qualcosa.

\section{In spregio o in sfregio? Qualche chiarimento in merito}

fregio e spregio sono due nomi antonimi (con s-privativa) di fregio e pregio e vanno in parallelo con i verbi sfregiare e spregiare, i quali a loro volta sono gli antonimi di fregiare e pregiare. Pregio significa qualità apprezzata (dal lat. pretium, da cui anche prezzo) e spregio (il cui allotropo è sprezzo), significa disprezzo. Fregio significa invece decorazione, ornamento, deonomastico da Phrigium, il nome degli abitanti della Frigia, a quanto pare cosi famosi per le decorazioni... pregiate (il loro re fu il mitico Mida!) da dare loro il nome. Il suo contrario, sfregio, significa non solo assenza di ornamento, ma anche sua deliberata cancellazione, deturpazione ("lo sfregio del vandalo ha rovinato il quadro"). Poiché fregio ha presto assunto anche il valore figurato di indizio di qualità morale, all'antonimo sfregio è toccato pure quello di denunciare la sua mancanza (per tacere dei casi in cui uno poteva antifrasticamente fare fregio di disvalori come il celebre Capaneo di Inferno XIV...) e il suo significato, favorito dalla somiglianza dei significanti, ha finito per sovrapporsi a quello di spregio ancor più di quanto potessero già sovrapporsi quelli di fregio e pregio (si pensi a espressioni come "Maria non ha partecipato alla tua festa per sfregio", cioè 'con l'intenzione di offenderti').

Due etimi assai diversi, due forme foneticamente non molto dissimili, due significati che finiscono per assomigliare. Lo sfregio si fa; lo spregio si ostenta, questa forse la differenza più forte tra i due sostantivi. Ma, tra i sensi figurati di sfregio e quelli propri di spregio, lo slittamento è facilissimo ed è stato comune, specie nelle locuzioni segnalate dai lettori. Di qui i comprensibili dubbi su di esse: $a /$ in spregio di e a/in sfregio di possono equivalersi per significato. In questo esempio da Riccardo Bacchelli riportato dal GDLI invece di sfregio si potrebbe anche avere spregio (e ancor meglio il suo allotropo sprezzo):

La loro cavalleria delittuosa li aveva spinti a mandar quei cinque a prender parte nei torbidi bolognesi, come in ogni altra braveria azzardata e pazza, tanto meglio se a sfregio d'ogni legge.

Questi altri, da Google Libri, ci ricordano bene l'intercambiabilità tra spregio e sprezzo:

Rifiutò la medaglia (datagli dal re) a spregio della maestà regia (G. De Sivo, Storia delle Due Sicilie, I863)

a sprezzo del comando Sovrano progredi a servire alle viste rivoluzionarie (G. Riccini, Appendice a completazione delle sue anteriori stampe, I851)

La forma storicamente più corretta è a spregio di ("a spregio del ridicolo", "a spregio del buon gusto") o in spregio di/a, nel senso di 'sprezzante, incurante di', 'senza badare a' ("in spregio al buon senso", "in/a 
spregio della morale comune"). La reggenza preferibile (rispondo a una delle domande) è di, ben attestata nel tempo e del resto richiesta da spregio ("lo spregio della vita", non "alla vita"), come dal parallelo sprezzo ("sprezzo della sorte" non "alla sorte").

Per completezza, segnaliamo che c'è anche il termine spreto (dal lat. spretum 'disprezzo'), di uso molto raro, che compare quasi esclusivamente nella locuzione (di sapore burocratico) in spreto a, nel senso di "non curandosi di' ("il bando del Comune ha fissato dei criteri in spreto alle leggi vigenti") o anche, genericamente, di "nonostante" ("il soprano, alla fine dell'opera, ha deluso il pubblico, in spreto a un promettente primo atto").

Se non è seguito da alcuna precisazione, il valore di sfregio (specie figurato) e di spregio è dunque negativo, indica, in sfregio, assenza o deliberata cancellazione di una qualità, e, in spregio, sprezzante negazione di essa. Per questo mi sembra strano (vengo a un'altra domanda) che si chieda un like " a sfregio" o anche "a spregio" del proprio intervento, perché sarebbe un gradimento di... disapprovazione, un ossimoro vero e proprio. L'utente di FB di cui parla il nostro lettore avrà voluto dire, suppongo, a fregio, cioè a chiosa di apprezzamento (come un fregio, insomma); a meno che il post non critichi qualcuno e quindi il like richiesto costituisca uno sfregio nei confronti di costui, e non del post.

Ma la valenza negativa di a spregio di ("a spregio dei santi" fa un modo di dire non elogiativo di colui cui è riferito) può essere compensata se segue un elemento a sua volta semanticamente negativo, che viene cosi negato dando all'insieme un valore positivo: "il prode si è tuffato a spregio del pericolo". Per la verità nel linguaggio delle menzioni che segnalano con lode un gesto di coraggio si legge spesso invece di "a spregio del pericolo" il suo allotropo "a sprezzo del pericolo", con significato identico.

Ci sono anche usi particolari di queste locuzioni. Nel linguaggio giuridico-burocratico, in spregio di vale in violazione di, ma è di uso raro (un po' meno del sopra citato in spreto a, sostanzialmente equivalente). Sul GDLI è registrata anche, da Bacchelli, la locuzione a sfregio di, col valore di 'a dispetto di, malgrado'; ma non sono né l'uno né l'altro troppo diversi da quelli già visti.

Riepilogando: le due forme, nelle locuzioni date, a/in sfregio di e a/in spregio di (cui è avvicinabile anchea sprezzo di), hanno significati simili, ben piu di quanto lo siano quelli di partenza di sfregio e di spregio ("lo sfregio di una statua" non è la stessa cosa del "suo spregio", ma "a sfregio delle buone maniere" non è troppo diverso da "a spregio delle stesse") e degli antonimi positivi fregio e pregio. La loro sovrapposizione quindi è comprensibile. Se si può, però, è meglio evitarla, ricorrendo alla forma più appropriata, a/in spregio di, quando non a quella più appropriata ancora di a sprezzo di. In Ermanno Rea (La dismissione 2002) si legge di "finestre distribuite in modo capriccioso e arbitrario, in spregio a ogni regola architettonica". Sarebbe stato non impossibile, ma meno appropriato, in sfregio di; sarebbe stato invece ancora più appropriato a sprezzo di.

Ergo, manteniamo le differenze tra sfregio e spregio, e non dimentichiamoci di sprezzo. Un exemplum fictum può essere utile per un chiarimento definitivo. Noi diremo che "in spregio (o a sprezzo) di ogni più elementare prudenza, Tizio è saltato dalla finestra ed è entrato nell'appartamento mettendo in salvo gli occupanti" e non che "in/a sfregio di ogni più elementare prudenza, Tizio ecc.", vero?

\section{Cita come:}

Vittorio Coletti, In spregio o in sfregio? Qualche chiarimento in merito , "Italiano digitale", 2019, X, 2019/3 (luglio-settembre)

DOI: $10.35948 / 2532-9006 / 2020.3193$ 